\title{
Stereoselective Production of Dimethyl-Substituted Carbapenams via Engineered Carbapenem Biosynthesis Enzymes
}

\author{
Refaat B. Hamed, ${ }^{\mathrm{a}, \mathrm{b}}$ Luc Henry, ${ }^{\mathrm{a}}$ Timothy D. W. Claridge, ${ }^{\mathrm{a}}$ and Christopher J. Schofield ${ }^{\mathrm{a} *}$

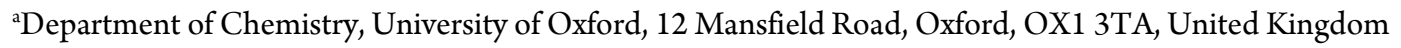 \\ ${ }^{\text {b} D e p a r t m e n t ~ o f ~ P h a r m a c o g n o s y, ~ F a c u l t y ~ o f ~ P h a r m a c y, ~ A s s i u t ~ U n i v e r s i t y, ~ A s s i u t, ~ 71526, ~ E g y p t ~}$ \\ *E-mail: christopher.schofield@chem.ox.ac.uk.
}

\begin{abstract}
Stereoselective biocatalysis by crotonase superfamily enzymes is exemplified by use of engineered 5-carboxymethylproline synthases (CMPSs) for preparation of functionalized 5-carboxymethylproline (5-CMP) derivatives methylated at two positions (i.e. C2/C6, C3/C6 and C5/C6), including products with a quaternary centre, from appropriately-substituted-amino acid aldehydes and C-2 epimeric methylmalonyl-CoA. The enzymatically-produced disubstituted 5-CMPs were converted by carbapenam synthetase into methylated bicyclic $\beta$-lactams, which manifest improved hydrolytic stability compared to the unsubstituted carbapenams. The results highlight the use of modified carbapenem biosynthesis enzymes for production of new carbapenams with improved properties.
\end{abstract}

KEYWORDS: stereoselective biocatalysis, protein engineering, crotonases, antibiotic biosynthesis, $\beta$-lactams

Although resistance to them is growing, the $\beta$-lactams remain the most important antibacterials. ${ }^{1}$ The carbapenems, which are often the most effective $\beta$-lactams from a clinical perspective, are produced by total synthesis, normally rendering them more expensive than penicillins and cephalosporins, which are derived from fermented products. This 'cost of goods' issue has limited clinical studies on carbapenem variants. Structure activity relationship (SAR) studies, on carbapenems ${ }^{2}$ and other $\beta$-lactam antibacterials, reveal that the nature and pattern of substitution of the bicyclic system modulates antibacterial activity, resistance to hydrolysis by $\beta$-lactamases and other enzymes (e.g. renal dehydropeptidases ${ }^{3-4}$ ), and non-enzymatic hydrolytic stability. ${ }^{5}$ We have thus been exploring the scope of engineered carbapenem biosynthesis enzymes for the biocatalytic synthesis of bicyclic $\beta$-lactam antibacterials, $\beta$-lactam based inhibitors of other targets, ${ }^{6-8}$ and of proline derivatives for use in catalysis. ${ }^{9-10}$

Three enzymes, CarB, CarA, and CarC, ${ }^{11-12}$ are directly responsible for the biosynthesis of the simplest carbapenem, $(5 R)$-carbapen-2em-3-carboxylate, in Pectobacterium carotovorum. In contrast multiple enzymes are involved in the assembly of the C2/C6-disubstituted carbapenem thienamycin in Streptomyces cattleya, making the engineering of this pathway more challenging (Fig. 1A). ${ }^{2,13}$ In both cases, a 5-membered $\beta$-amino acid carbapenam precursor is initially constructed as catalysed by a member of the crotonase superfamily: ${ }^{14}$ CarB in P. carotovorum ${ }^{15-18}$ and ThnE in $S$. cattleya. ${ }^{19-20}$ These crotonases catalyse the selective transformation of malonyl$\mathrm{CoA}$ and an equilibrating mixture of $(2 S)$-glutamate semialdehyde/5-hydroxyproline/pyroline-5-carboxylate (collectively $(2 S$ )GHP) into (2S,5S)-5-carboxymethylproline ( $t$-CMP)) (Fig. 1A). CarB/ThnE catalysis proceeds via malonyl-CoA decarboxylation to give an enzyme-bound acetyl-CoA enolate that is likely stabilized by an oxy-anion hole, which is formed by two residues in the active site of the carboxymethylproline synthases (CMPSs) (Fig. 1B). C-C bond formation then proceeds via nucleophilic attack of the enolate on the $(R e)$-face of the iminium form of pyroline-5-carboxylate.
Subsequent hydrolysis of the resultant thioester intermediate gives $t$-CMP and CoASH (Fig. 1A). ${ }^{15-16}$ The ATP and $\mathrm{Mg}^{\mathrm{II}}$-dependent $\beta$ lactam synthetase CarA in $P$. carotovorum (and likely ThnM in $S$. cattleya) then catalyzes cyclisation of $t$-CMP into $(3 S, 5 S)$-carbapenam-3-carboxylate $((3 S, 5 S)-\mathrm{C} 3 \mathrm{C})$, Fig. $1 \mathrm{~A}^{20-21}$ While the enzyme(s) catalyzing C-5 epimerization and C-2/C-3 desaturation are yet to be defined in thienamycin biosynthesis; ${ }^{2}$ in $P$. carotovorum, the 2-oxoglutarate and $\mathrm{Fe}^{\mathrm{II}}$-dependent oxygenase CarC catalyzes formation of $(5 R)$-carbapen-2-em-3-carboxylate from $(3 S, 5 S)-\mathrm{C} 3 \mathrm{C}$ (Fig. 1A). ${ }^{22-23}$

We have described the use of CarB and ThnE variants to catalyse production of singly alkylated $t$-CMP derivatives, at either C-2 or C6 from the appropriate $(2 S)$-GHP and malonyl-CoA derivatives. ${ }^{15,24}$

${ }^{26}$ We now report on the use of engineered CMPSs to catalyse the stereoselective preparation of disubstituted CMP derivatives, including some with a quaternary carbon, from appropriately-substituted amino-acid aldehydes and C-2 epimeric methylmalonyl-CoA. The potential utility of the method is demonstrated by conversion of the products into bicyclic $\beta$-lactams using CarA catalysis.

Preparation of (2,6)-dimethyl- $t$-CMP derivatives: We began by testing the capacity of reported CMPS variants ${ }^{24-28}$ to catalyse formation of 2,6-dimethyl-t-CMP derivatives (Scheme 1I). The selection of CMPS variants for testing for production of dimethylated- $t$-CMP derivatives was guided by earlier studies revealing that certain CMPS variants manifest relaxed substrate selectivity, e.g. CarB H229A for construction of 2-methyl-substituted $t$-CMP derivatives. ${ }^{25}$

Incubation of racemic $(2 S / 2 R)$-2-methyl-GHP ${ }^{25}$ and $\mathrm{C}-2$ epimeric methylmalonyl-CoA with CarB H229A (the highest yielding CMPS variant identified for this transformation), followed by LC-MS analysis resulted in observation of two species, both with the predicted mass for the epimeric dimethylated products $(\mathrm{m} / \mathrm{z}=202.1$ $\left.[\mathrm{M}+1]^{+}\right)$. The ratio between the two C- 6 epimers observed was 63:37 (as observed by LC-MS and NMR analyses). Scale-up and LC-MS guided purification led to the isolation ( $>95 \%$ pure by ${ }^{1} \mathrm{H}-$ 


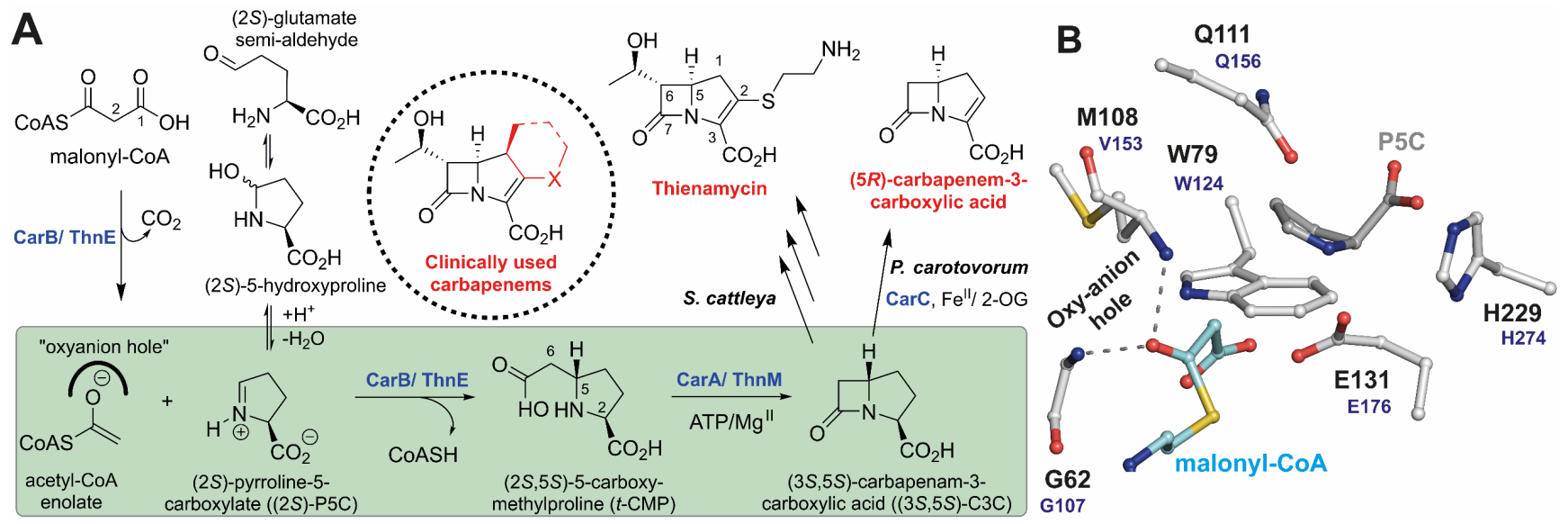

Figure 1. Carbapenem biosynthesis. A: Roles of the enzymes utilized in this study in the biosynthesis of (5R)-carbapenem-3-carboxylic acid (i.e. CarB and CarA in Pectobacterium carotovorum) and thienamycin (i.e. ThnE, in Streptomyces cattleya). ${ }^{2}$ Note that many clinically used carbapenems are substituted at C-1 and are always substituted at C-2 and C-6, sometimes forming a tricyclic system (insert, X = sulfur or carbon); B: View from a crystal structure of $\mathrm{CarB}^{18}$ with malonyl-CoA and (2S)-pyrroline-5-carboxylate (P5C) modeled into the active site, highlighting some of the active site residues, which were substituted in the variants used in this study (the analogous ThnE residues are shown in blue). ${ }^{19}$

NMR, Fig. 2A and B) of the two C-6 epimers that were characterised by $2 \mathrm{D}$ NMR analyses (Figs. S1 and S2). The NMR analyses revealed the first and second eluting epimers to have the $(2 S, 6 S)$ and $(2 S, 6 R)$-stereochemistry, respectively. In contrast, with the available set of CMPS variants (Table S1), use of C-2 epimeric ethylmalonyl-CoA ${ }^{19}$ and 2-methyl-GHP did not result in detectable $t$-CMP derivatives (by LC-MS analysis). Notably, the other CarB/ThnE variants tested (Table S1) were unable to catalyse formation of the 2,6-dimethyl- $t$-CMP epimers at appreciable levels ( $<5 \%$ by LC-MS analysis).
These results demonstrate the ability of CarB H229A to catalyse formation of 2,6-dimethyl-t-CMP derivatives with adjacent tertiary stereocentres (at C5 and C6), in addition to the preinstalled and sterically-demanding quaternary centre (at C2), in a single transformation and in moderate unoptimised isolated yield (28\% from racemic $(2 S / 2 R)$-2-methyl-GHP, Table 1 , entry 1$)$. The capacity of CarB H229A to accept substrate analogues with a quaternary C-2 stereocentre is likely due to increased active site volume in the region binding $\mathrm{C}-2$ as implied by crystallographic studies $^{18}$ (Fig. 1B).

Scheme 1. Products of CMPS catalysis resulting from incubation of C2-, C3-, and C5-methylated-(2S)-GHP derivatives with C-2 epimeric methylmalonyl-CoA.

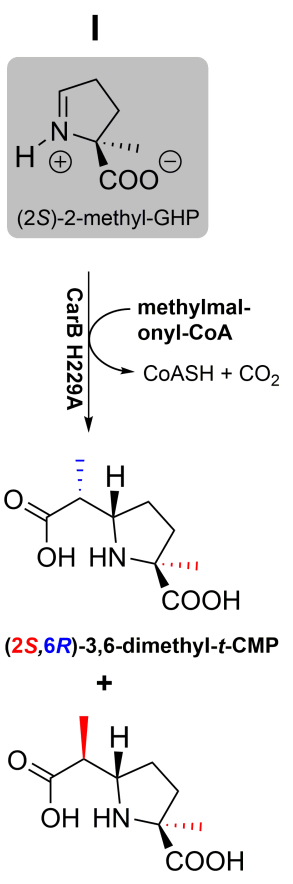

(2S,6S)-3,6-dimethyl-t-CMP
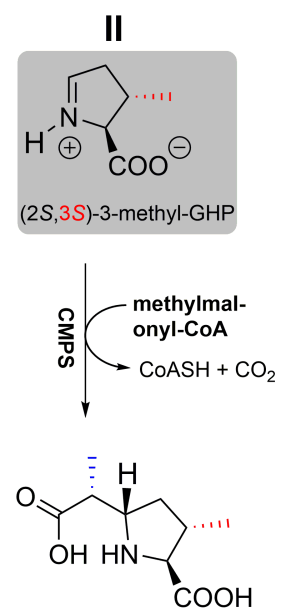

(3S,6R)-3,6-dimethyl-t-CMP

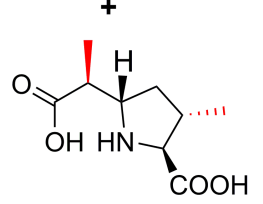

(3S,6S)-3,6-dimethyl-t-CMP
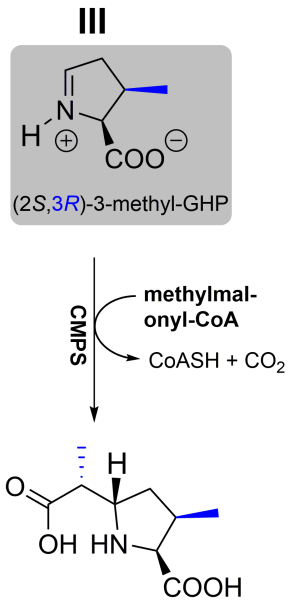

(3R,6R)-3,6-dimethyl-t-CMP

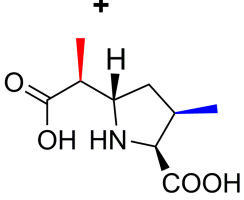

(3R,6S)-3,6-dimethyl-t-CMP
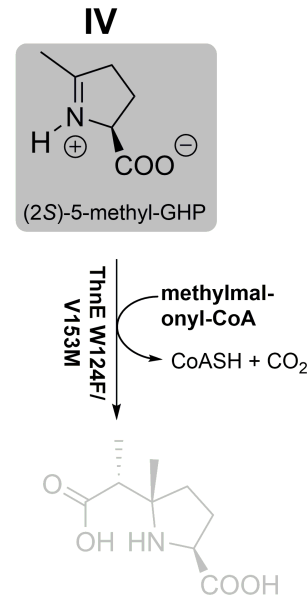

$(5 S, 6 R)-5,6$-dimethyl-t-CMP

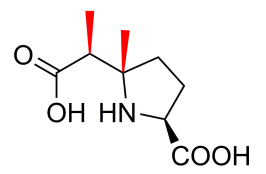

(5S,6S)-5,6-dimethyl-t-CMP

A potential product that was not observed, within limits of detection, is in grey. The GHP derivatives (in the case of I, II and III) were prepared as racemates, but only compounds with the $(2 S)$-stereochemistry are known to be CMPS susbtrates. ${ }^{19,25,29}$ 


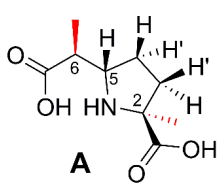

(2S,6S)-2,6-dimethyl$t$-CMP

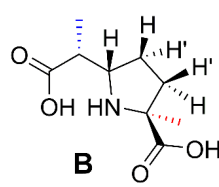

(2S,6R)-3,6-dimethyl$t$-CMP

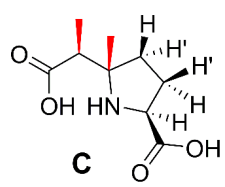

(5S,6S)-3,6-dimethyl$t$-CMP

$\mathrm{H} 2$
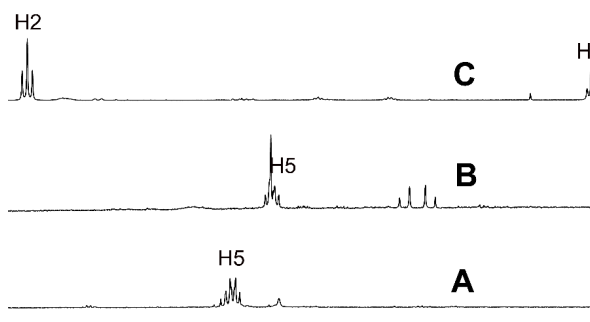

4.0

"s

wher

3.5

B

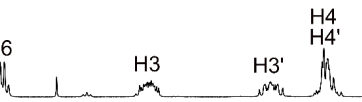

Figure 2. Production of dimethyl-substituted-t-CMP derivatives with quaternary stereocentres. A, B and C: ${ }^{1} \mathrm{H}-\mathrm{NMR}$ spectra for the shown dimethylsubstituted- $t$-CMP epimers resulting from incubation of C-2 epimeric methylmalonyl-CoA and racemic 2-methyl-GHP (A and B)/(2S)-5-methylGHP (C), as catalyzed by CarB H229A (A and B)/ThnE W124F/V153M (C), respectively; D: View from a model of ThnE V153A variant active site based on structures of wildtype $\mathrm{CarB}^{18}$ with $(R)$-methylmalonyl-CoA and $(2 S)$-5-methyl-P5C substrate analogues modelled at the active site. The selectivity of ThnE V153A variant for production of the $(5 S, 6 S)$-5,6-dimethyl-t-CMP epimer can be rationalized by a steric clash between the methyl group of the $(Z)$-enolate resulting from decarboxylation of $(S)$-methylmalonyl-CoA and the methyl group of $(2 S)$-5-methyl-P5C. during the C-C bond formation; E: View from a ThnE model with $(R)$-methylmalonyl-CoA and $(2 S)$-P5C substrate in the active site. Note the potential for a steric clash between the methyl group of methylmalonyl-CoA and Val-153, which rationalizes preferential production of the $(6 R)$ - epimers by ThnE and ThnE variants with a $\beta$-branched amino acid at residue-153 (e.g. ThnE V153I). Note that the lack of such clash in $\mathrm{D}$ rationalizes for the preferential production of epimers with $(6 S)$-stereochemistry by ThnE and ThnE variants without a $\beta$-branched amino acid at position 153 (e.g. ThnE V153A).

Preparation of $(3,6)$-dimethyl-t-CMP derivatives: We then investigated preparation of 3,6-dimethyl- $t$-CMP derivatives, because many natural and synthetic carbapenems are substituted at bicyclic ring positions analogous to $\mathrm{C}-3$ and $\mathrm{C}-6$ of $t$-CMP (Figure $1 \mathrm{~A}){ }^{2}$ Incubation of $\mathrm{C}-2$ epimeric methylmalonyl-CoA and a racemic mixture of $(2 S, 3 S)-/(2 R, 3 R)$-3-methyl-GHP (only GHP substrates with the $(2 S)$, but not $(2 R)$-, stereochemistry are accepted by CarB, ThnE and variants thereof) ${ }^{19,25,29}$ with wildtype or engineered CMPS variants resulted in the observation (by LC-MS) of two products with the anticipated mass $\left(\mathrm{m} / z=202.1[\mathrm{M}+1]^{+}\right)$ (Fig. 3, Scheme 1II). Interestingly, the ThnE/ThnE-variants manifested different patterns of stereoselectivity towards production of the two observed C- 6 epimers of 3,6-dimethyl- $t$-CMP, compared to the CarB/CarB-variants (Fig. 3, Table S2). Wildtype CarB produced the highest relative yield of the first eluting epimer $(31 \%$, Table S2, entry 1); however, wildtype ThnE (Table S2, entry 2) and the ThnE V153I variant (Table 1, entry 2 ) produced the highest diastereomeric excess (d.e.) of the same epimer (d.e. $=0.86$, in the case of the ThnE V153I variant). Whilst the ThnE V153L variant produced the highest relative yield of the later-eluting epimer ( 42\%, Table 1, entry 3 ), the ThnE V153A variant produced the highest d.e. of the same epimer with a comparative yield (d.e., in the case of ThnE V153A, $=0.80$, Table S2, entry 9). Following LCMS-guided purification of the scaled-up preparation, NMR investigations of the major product of ThnE V153I catalysis revealed its stereochemistry as $(2 S, 3 S, 6 R)$ (Figs. 3, S3 and S4, assuming conversion of the $(2 S)$-substrate). ${ }^{19,25,29}$ The major product of ThnE V153L catalysis was shown to be the $(2 S, 3 S, 6 S)$-stereoisomer (Figs. 3, S3 and S5). The comparatively low yields in the case of ThnE W124F, ThnE W124F/V153M, and ThnE H274A variants (Table S2, entries 4-6, respectively) may reflect a slower rate of hydrolysis of the $t$-CMP-CoA thioester intermediate by these vari- ants (Fig. S6). Preliminary evidence for the slower rate of such hydrolysis originates from the observation of a species with $\mathrm{m} / \mathrm{z}=$ 216.1 (Figs. S6A and C), in the total ion chromatograms of these variants, corresponding to the methyl ester of 3,6-dimethyl- $t$ CMP, which we have proposed to be produced by methanolysis of the (normally enzyme-bound) thioester intermediate which leaks from the active site ${ }^{16}$ before or during methanol quenching of the reaction (Fig. S6D). ${ }^{24}$

Incubation of racemic $(2 S, 3 R)-/(2 R, 3 S)$-3-methyl-GHP ${ }^{25}$ and C2 epimeric methylmalonyl-CoA with wildtype and engineered CMPS variants resulted in the observation of two LC-MS peaks with the anticipated mass $\left(m / z=202.1[\mathrm{M}+1]^{+}\right.$) (Fig. 3, Scheme 1III and Table S3). Whilst CarB M108I produced the highest relative yield and d.e. of the first epimer to elute $(\sim 29 \%$ at d.e. $=0.06$, Table 1, entry 4), ThnE V153L (Table S3, entry 8) and CarB W79A (Table 1, entry 5) produced the highest relative yield and diastereomeric excess of the later-eluting epimer $(\sim 44 \%$ at d.e. $=$ 0.94, in the case of CarB W79A). Subsequent analyses identified the major product of CarB M108I catalysis as the $(2 S, 3 R, 6 R)$-stereoisomer (Figs. 3 and S7) and the major product of CarB W79A catalysis as the $(2 S, 3 R, 6 S)$-stereoisomer (Figs. 3 and S8). Note that the stereochemical assignments of disubstituted- $t$-CMP monocycles are supported by analyses on the corresponding bicyclic $\beta$ lactams (see below).

Incubation of $\mathrm{C}-2$ epimeric ethylmalonyl- $\mathrm{CoA}^{19}$ with either of the (2S)-3-methyl-GHP isomers did not result in the formation of $t$ CMP derivatives at appreciable ( $>5 \%)$ levels with any of the CMPSs tested, except for the CarB W79F and CarB W79A variants. LC-MS analyses revealed that these variants catalyse the formation of 6-ethyl-3-methyl- $t$-CMP diastereomers in yields ranging from 6 to 14\% (Fig. S9). The strong preference of the CarB W79A 
Table 1. $N$-Heterocycles resulting from incubation of C-2, C-3 and C-5 methylated-( $2 S)$-GHP with C-2 epimeric methylmalonyl-CoA as catalysed by the preferred engineered CMPS variants, in terms of yield and/or diastereomeric excess.

\begin{tabular}{|c|c|c|c|c|c|}
\hline & $\begin{array}{c}\text { C-2 epimeric } \\
\text { methylmalonyl-CoA }\end{array}$ & $\begin{array}{c}O \\
\text { methylated-(2S)-GHP }\end{array}$ & disubstitt & $\begin{array}{l}\mathrm{CO}_{2} \mathrm{H} \\
\mathrm{CMP}\end{array}$ & \\
\hline Entry & Catalyst & $(2 S)$-GHP Substrate & Product(s) & d.r. ${ }^{a}$ & \%Yield ${ }^{\mathrm{b}}$ \\
\hline \multirow[t]{2}{*}{1} & CarB H229A & $\mathrm{R}^{1}=\mathrm{CH}_{3}$ & $\mathrm{R}^{1}=\mathrm{R}^{5}=\mathrm{CH}_{3}$ & 63 & $14(28)$ \\
\hline & & & $\mathrm{R}^{1}=\mathrm{R}^{6}=\mathrm{CH}_{3}$ & 37 & \\
\hline \multirow[t]{2}{*}{2} & ThnE V153I & $\mathrm{R}^{2}=\mathrm{CH}_{3}$ & $\mathrm{R}^{2}=\mathrm{R}^{6}=\mathrm{CH}_{3}$ & 93 & $12(23)^{b, c}$ \\
\hline & & & $\mathrm{R}^{2}=\mathrm{R}^{5}=\mathrm{CH}_{3}$ & 07 & \\
\hline \multirow[t]{2}{*}{3} & ThnE V153L & $\mathrm{R}^{2}=\mathrm{CH}_{3}$ & $\mathrm{R}^{2}=\mathrm{R}^{6}=\mathrm{CH}_{3}$ & 14 & $25(49)^{b, c}$ \\
\hline & & & $\mathrm{R}^{2}=\mathrm{R}^{5}=\mathrm{CH}_{3}$ & 86 & \\
\hline \multirow[t]{2}{*}{4} & CarB M108I & $\mathrm{R}^{3}=\mathrm{CH}_{3}$ & $\mathrm{R}^{3}=\mathrm{R}^{6}=\mathrm{CH}_{3}$ & 53 & $27(54)^{b, c}$ \\
\hline & & & $\mathrm{R}^{3}=\mathrm{R}^{5}=\mathrm{CH}_{3}$ & 47 & \\
\hline \multirow[t]{2}{*}{5} & CarB W79A & $\mathrm{R}^{3}=\mathrm{CH}_{3}$ & $\mathrm{R}^{3}=\mathrm{R}^{6}=\mathrm{CH}_{3}$ & 03 & $22(45)^{b, c}$ \\
\hline & & & $\mathrm{R}^{3}=\mathrm{R}^{5}=\mathrm{CH}_{3}$ & 97 & \\
\hline 6 & ThnE W124F/V153M & $\mathrm{R}^{4}=\mathrm{CH}_{3}$ & $\mathrm{R}^{4}=\mathrm{R}^{5}=\mathrm{CH}_{3}$ & $>99: 1$ & 17 \\
\hline
\end{tabular}

${ }^{\text {a }}$ d.r.= diastereomeric ratio of C-6 epimers of $t$-CMP derivatives with a methyl substituent at C-2, C-3 and C-5, as determined by ${ }^{1} \mathrm{H}$ NMR spectroscopy and/or LC-MS analyses, under standard incubation conditions. ${ }^{b}$ The $\%$ yield (isolated, rounded numbers) was calculated over the following steps: ${ }^{25-}$ ${ }^{26,28}$ deprotection of amino acid aldehydes, incubation with enzyme(s), LC-MS (double) purification, and (double) lyophilization; products were quantified by ${ }^{1} \mathrm{H}$ NMR using $\left[{ }^{2} \mathrm{H}\right]_{4}$-trimethylsilylpropionate as a standard. ${ }^{\mathrm{c}}$ Starting materials in the case of entries 1-5 were prepared as racemates; yields, taking this into account, are in parentheses. $\mathrm{R}=\mathrm{H}$ may be assumed, unless otherwise stated.<smiles>[B]C(C(=O)O)[C@H]1C[C@H](C)[C@@H](C(=O)O)N1</smiles>

$(3 S, 6 R)-3,6$-dimethyl-t-CMP $\quad(3 S, 6 S)-3,6-$ dimethyl-t-CMP

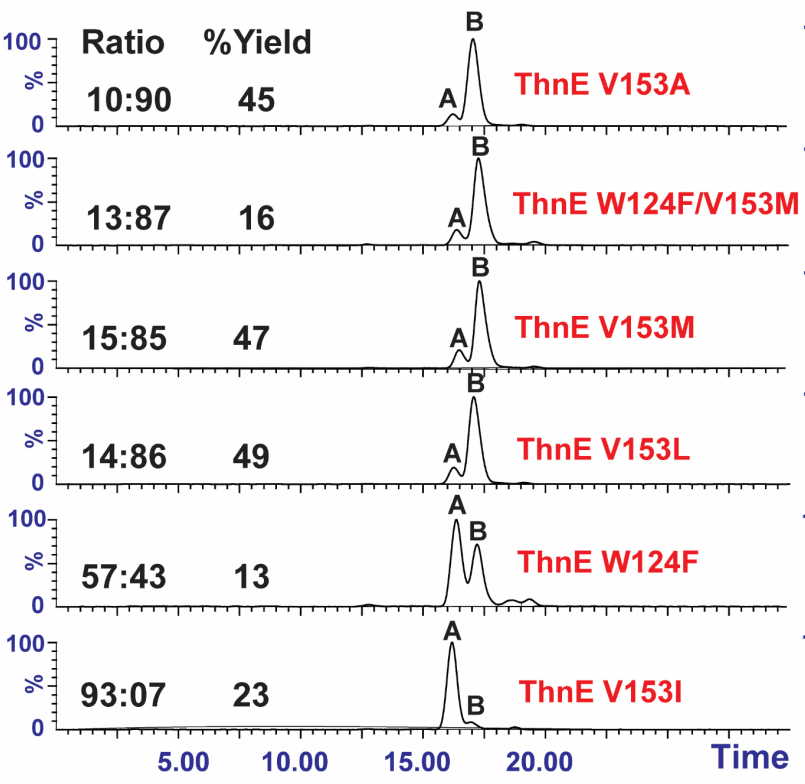<smiles>C[C@H]1C[C@H](C)[C@H](C(=O)O)N1</smiles>

$(3 R, 6 R)-3,6$-dimethyl-t-CMP<smiles>[B]C(C(=O)O)[C@H]1C[C@H](C)[C@@H](C(=O)O)N1</smiles>

$(3 R, 6 S)-3,6$-dimethyl-t-CMP

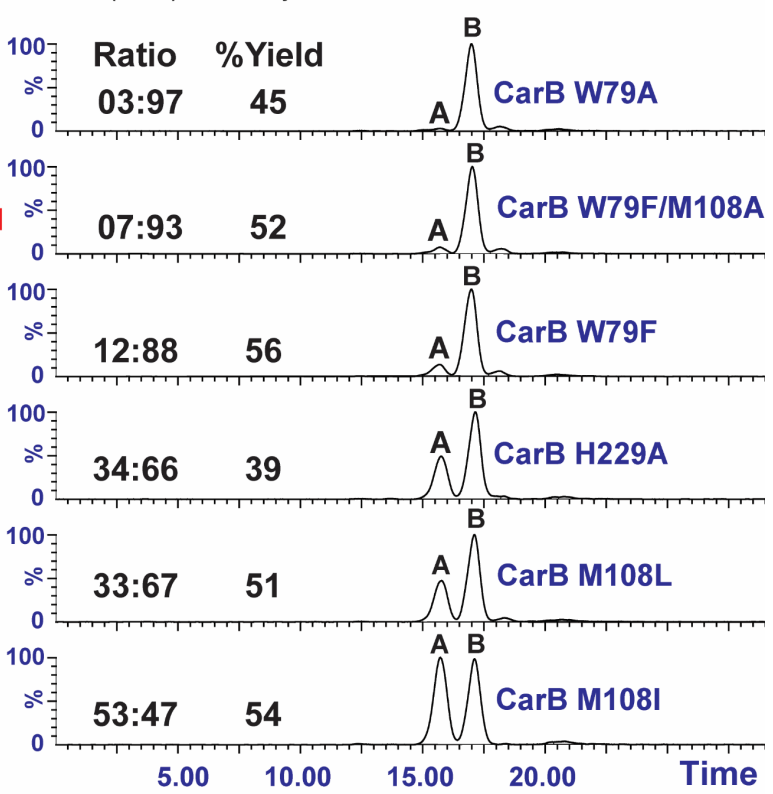

Figure 3. Ion-extracted LC-MS chromatograms (ESI+, $m / z=202.1$ ), yields and diastereomeric ratios for the two C-6 epimers of (3S)-3,6-dimethyl- $t$ CMP (left) and those of (3R)-3,6-dimethyl-t-CMP (right) obtained by incubation of C-2 epimeric methylmalonyl-CoA and racemic (2S,3S)-3-methyl-GHP or racemic $(2 S, 3 R)$-3-methyl-GHP, respectively, as catalysed by CMPS variants, under standard conditions. Only the preferred engineered CMPS variants, in terms of yield and/or diastereomeric excess, are presented. 
variant for products with the $(6 S)$-stereochemistry was largely maintained when racemic $(2 S, 3 R)$-3-methyl-GHP and C-2 epimeric ethylmalonyl-CoA were used as co-substrates (d.e. $=0.94$, Fig. S9). Coupling of crotonyl-CoA carboxylase/reductase (Ccr) catalysis, which exclusively produces $(2 S)$-ethylmalonyl-CoA, ${ }^{30}$ to that of CarB W79F, ${ }^{24,26}$ was employed to conclusively assign the C6 stereochemistry of the resulting 6-ethyl-3-methyl- $t$-CMP diastereomers (Fig. S10).

Preparation of $(5,6)$-dimethyl-t-CMP derivatives: Many alkaloids contain a substructure comprising a 5-membered $\mathrm{N}$-heterocycle with a quaternary centre, and possess a wide range of biological activities (e.g. physostigmine). ${ }^{31}$ The possibility of stereocontrol by CMPS catalysis with adjacent stereocentres was thus evaluated employing C-5 epimeric (2S)-5-methyl-GHP (i.e. a mixture of $(2 S, 5 R)$ - and (2S,5S)-5-methyl-GHP) and C-2 epimeric methylmalonyl-CoA (Scheme 1IV). LC-MS analyses revealed a single chromatographic peak with the anticipated mass $(\mathrm{m} / \mathrm{z}$ $\left.=202.1[\mathrm{M}+1]^{+}\right)$, as catalysed by wild-type ThnE, ThnE V153A or ThnE W124F/V153M only (Fig. S11).

ThnE W124F/V153M was the highest yielding variant; the relative yields of wild-type ThnE and ThnE V153A were $6 \%$ and $68 \%$, respectively (Fig. S11). Following, scaled-up preparation, guided by LC-MS, 2D NMR analysis of the product of ThnE $\mathrm{W} 124 \mathrm{~F} / \mathrm{V} 153 \mathrm{M}$-catalysis revealed that the product formed is exclusively the $(2 S, 5 S, 6 S)$-5,6-dimethyl-CMP stereoisomer, with no evidence for formation of any other of the three possible stereoisomers, including the $(2 S, 5 S, 6 R)$ epimer (Scheme $1 I V$, Table 1 , entry 6, Figs. 1, S12 and S13). When C-2 epimeric ethylmalonyl-CoA was incubated with $(2 S)$-5-methyl-GHP, no CMP derivative was detected, by LC-MS, with any of the tested CMPSs.

Overall, these results reveal the capacity of CMPS variants to catalyse the stereocontrolled generation of contiguous, tertiary and heteroatom-substituted quaternary, stereocentres in a single transformation (in $\sim 17 \%$ isolated yield and $>0.99$ d.e. in the case of ThnE W124F/V153M).

Conversion of $t$-CMP derivatives into bicyclic $\beta$-lactams: We then investigated conversion of the dimethyl-substituted- $t$-CMP derivatives to bicyclic $\beta$-lactams using the synthetase CarA (Fig. 1A). CarA efficiently catalysed the conversion of five out of the seven prepared dimethyl-substituted- $t$-CMP derivatives tested (75$100 \%$ ), as demonstrated by LC-MS and (2D) NMR analyses (Table 2 and Figs. 4, S14-S22). The results reveal that dimethyl-substituted- $t$-CMP diastereoisomers with the $(6 S)$-stereochemistry are less favored CarA substrates compared to the $(6 R)$-epimers (compare entries 1, 3 and 5 with entries 2, 4, 6 and 7, Table 2), likely due to steric constraints (Fig. 4D/E). Indeed, in the cases of two disubstituted- $t$-CMP derivatives with the $(6 S)$-stereochemistry (i.e. $(2 S, 6 S)$-2,6-dimethyl-t-CMP and $(5 S, 6 S)$-5,6-dimethyl- $t$ CMP), no evidence for CarA catalysed bicyclisation was accrued, under the tested conditions (Table 2, entry 2 and 7 ). The conversion of the reported $t$-CMP derivatives to bicyclic $\beta$-lactams enabled more definitive assignment of the C- 6 stereochemistry, which is difficult in the monocyclic $t$-CMP derivatives due to rotation about the C5-C6 bond. Whilst diastereomers with the $(6 S)$-stereochemistry manifested a $J_{5,6}=1.6-1.8 \mathrm{~Hz}$, which is typical for $\beta$ lactams with $\mathrm{H} 5$ and $\mathrm{H} 6$ in a trans-relationship (Figs. S20 and $\mathrm{S} 22$ ), those with the $(6 R)$-stereochemistry manifested a $J_{5,6}=4.8$ -

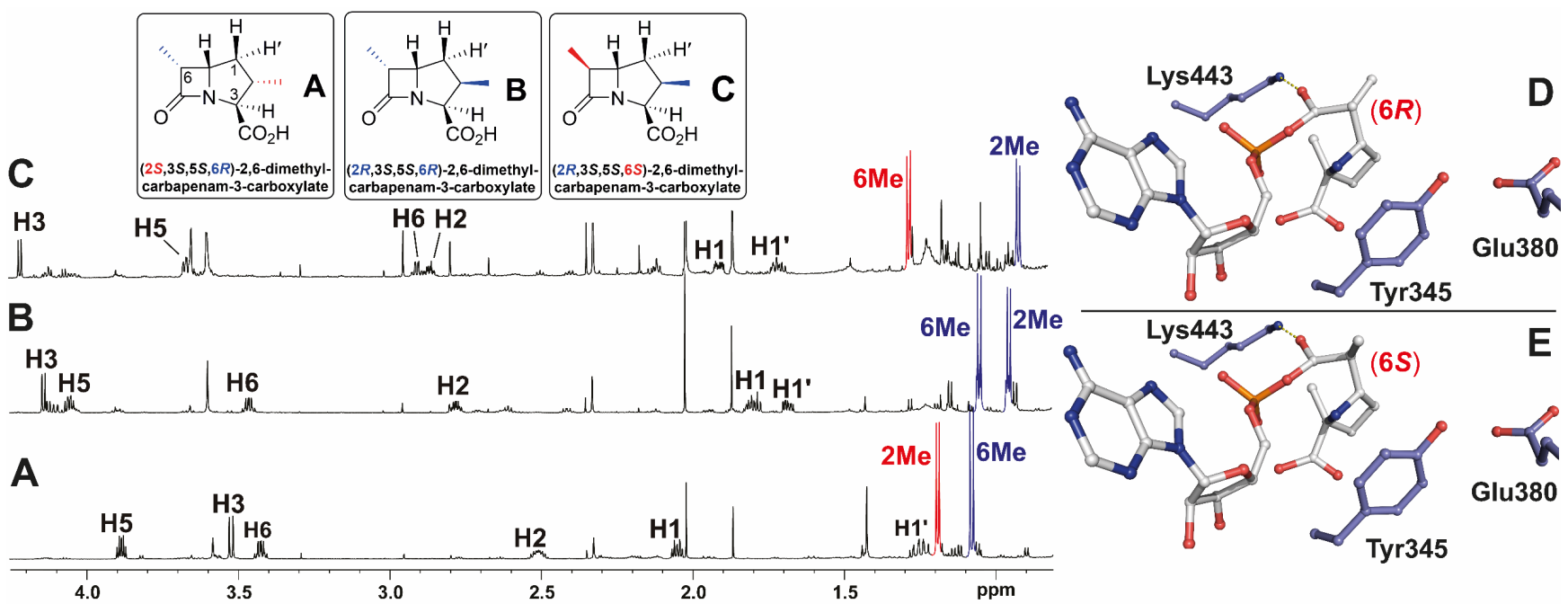

Figure 4. Preparation of dimethyl-substituted carbapenam-3-carboxylic acids by CarA catalysis. A-C: ${ }^{1} \mathrm{H}$ NMR spectra of the 3,6-dimethyl-substituted carbapenam-3-carboxylic acids prepared from 3,6-dimethyl-t-CMP derivatives (Table 2) employing CarA (For more examples, see Figs. S15 and S20); D and E: Views from a CarA crystal structure (PDB 1Q19) ${ }^{32}$ with the acyl-adenylate intermediate of the C-6 epimers of 2,6-dimethyl- $t$-CMP modelled in the active site. Note that the intramolecular Bürgi-Dunitz trajectory for nucleophilic attack of the secondary amine on the carbonyl of the acyladenylate appears less sterically hindered for the $(6 R)$-epimer $(\mathbf{A})$ compared to the $(6 S)$-epimer $(\mathbf{B})$, rationalizing the preference of CarA for substrate epimers with the $(6 R)$-stereochemistry. In both cases, the fold of the acyl-adenylate intermediate is likely to suffer limited conformational space because of the bulky substituent at C-2 of the $t$-CMP nucleus. The model is built on the basis of the acyl-adenylate $N^{2}$-(2-carboxymethyl)arginine-AMP species bound in a crystal structure of $\beta$-LS (PDB 1MBZ). ${ }^{33}$ The Lys443 residue is proposed ${ }^{34}$ to assist in ring cyclisation via stabilisation of the proposed "tetrahedral" intermediate (resulting upon nucleophile attack by the secondary amine of $t$-CMP). The shown Tyr345-Glu380 dyad is proposed to deprotonate the amine involved in the intramolecular $\beta$-lactam formation. ${ }^{35}$ 
Table 2. Conversion of disubstituted-t-CMP derivatives into disubstituted-carbapenam-3-carboxylate (C3C) derivatives by carbapenam synthetase ( Car A) catalysis.

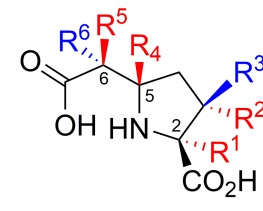

disubstituted-t-CMP

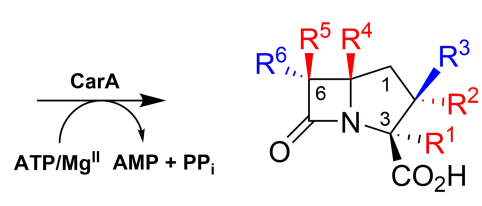

disubstituted-carbapenam3-carboxylate (C3C)

\begin{tabular}{|c|c|c|c|c|}
\hline Entry & Substrate & Product & $\%$ Conversion $^{\mathrm{a}}$ & $t_{1 / 2}(\text { days })^{\mathrm{b}}$ \\
\hline 1 & $\begin{array}{l}(2 S, 6 R) \text {-2,6-dimethyl- } t \text {-CMP: } \\
\mathrm{R}^{1}=\mathrm{R}^{6}=\mathrm{CH}_{3}\end{array}$ & $\begin{array}{l}(3 S, 5 S, 6 R) \text {-3,6-dimethyl-C3C: } \\
\mathrm{R}^{1}=\mathrm{R}^{6}=\mathrm{CH}_{3}\end{array}$ & 75 & \\
\hline 2 & $\begin{array}{l}(2 S, 6 S) \text {-2,6-dimethyl- } t \text {-CMP: } \\
\mathrm{R}^{1}=\mathrm{R}^{5}=\mathrm{CH}_{3}\end{array}$ & $\begin{array}{l}(3 S, 5 S, 6 S) \text {-3,6-dimethyl-C3C: } \\
\mathrm{R}^{1}=\mathrm{R}^{5}=\mathrm{CH}_{3}\end{array}$ & 0 & \\
\hline 3 & $\begin{array}{l}(3 S, 6 R)-3,6 \text {-dimethyl- } t \text {-CMP: } \\
\mathrm{R}^{2}=\mathrm{R}^{6}=\mathrm{CH}_{3}\end{array}$ & $\begin{array}{l}(2 S, 3 S, 5 S, 6 R)-2,6 \text {-dimethyl-C3C: } \\
\mathrm{R}^{2}=\mathrm{R}^{6}=\mathrm{CH}_{3}\end{array}$ & 100 & 16 \\
\hline 4 & $\begin{array}{l}(3 S, 6 S)-3,6 \text {-dimethyl- } t \text {-CMP: } \\
\mathrm{R}^{2}=\mathrm{R}^{5}=\mathrm{CH}_{3}\end{array}$ & $\begin{array}{l}(2 S, 3 S, 5 S, 6 S) \text {-2,6-dimethyl-C3C: } \\
\mathrm{R}^{2}=\mathrm{R}^{5}=\mathrm{CH}_{3}\end{array}$ & 83 & 24 \\
\hline 5 & $\begin{array}{l}(3 R, 6 R)-3,6 \text {-dimethyl- } t \text {-CMP: } \\
\mathrm{R}^{3}=\mathrm{R}^{6}=\mathrm{CH}_{3}\end{array}$ & $\begin{array}{l}(2 R, 3 S, 5 S, 6 R)-2,6 \text {-dimethyl-C3C: } \\
\mathrm{R}^{3}=\mathrm{R}^{6}=\mathrm{CH}_{3}\end{array}$ & 100 & \\
\hline 6 & $\begin{array}{l}(3 R, 6 S) \text {-3,6-dimethyl- } t \text {-CMP: } \\
\mathrm{R}^{3}=\mathrm{R}^{5}=\mathrm{CH}_{3}\end{array}$ & $\begin{array}{l}(2 R, 3 S, 5 S, 6 S) \text {-2,6-dimethyl-C3C: } \\
\mathrm{R}^{3}=\mathrm{R}^{5}=\mathrm{CH}_{3}\end{array}$ & 87 & \\
\hline 7 & $\begin{array}{l}(5 S, 6 S) \text {-2,6-dimethyl-t-CMP: } \\
\mathrm{R}^{4}=\mathrm{R}^{5}=\mathrm{CH}_{3}\end{array}$ & $\begin{array}{l}(3 S, 5 S, 6 S)-5,6 \text {-dimethyl-C3C: } \\
\mathrm{R}^{4}=\mathrm{R}^{5}=\mathrm{CH}_{3}\end{array}$ & 0 & \\
\hline
\end{tabular}

a $\%$ conversion was determined by LC-MS analyses. ${ }^{\mathrm{b}}$ The time required for half of the carbapenam to decompose; calculated for the shown derivatives only, where the purified carbapenam was kept (inside a $2 \mathrm{~mm} \mathrm{NMR}$ tube) at $4^{\circ} \mathrm{C}$ in sodium formate $\mathrm{pH} 7$ (as described in the supporting information). Hydrolysis was monitored by ${ }^{1} \mathrm{H}$ NMR analyses (e.g., see Fig. S23). $\mathrm{R}=\mathrm{H}$ may be assumed, unless otherwise stated.

$5.3 \mathrm{~Hz}$, which is typical for $\beta$-lactams with $\mathrm{H} 5$ and $\mathrm{H} 6$ in a cis-relationship (Figs. S15, S18 and S21). ${ }^{36}$ The simplest (unsubstituted) carbapenem, (5R)-carbapenem-3-carboxylate (Fig. 1A) is highly unstable, to the extent that its isolation in the free form (rather than as ester derivative) has not been possible. ${ }^{37}$ Indeed only five carbapenams ${ }^{2}$ have been isolated from natural sources, mainly as ester derivatives, likely due to instability of the free acids. ${ }^{38-41}$ Interestingly, some of the disubstituted carbapenams prepared using CarA displayed increased resistance to hydrolysis $\left(t_{1 / 2} \sim 16-24\right.$ days, $4^{\circ} \mathrm{C}$, in sodium formate buffer $\mathrm{pH} \sim 7$; Table 2 , entry 3 and 4 , and Fig. S23) when compared to their unsubstituted/mono-substituted counterparts, which, in our hands, almost completely hydrolyzed during post-purification lyophilization, as evidenced by NMR analyses.

Discussion: The overall results illustrate the biocatalytic scope of CMPS variants for the stereoselective synthesis of dialkylated- $t$ CMP derivatives; such compounds are of interest as antibacterial precursors and as starting materials for the preparation of $\beta$-lactams with other targets, as well as for proline-related organic catalysts. The combined use of the CMPS variants and CarA enables the two-step synthesis of densely functionalized bicyclic $\beta$-lactams from alkyl-malonyl-CoA and (2S)-GHP derivatives. The cost of reagents means the present conditions are unlikely to be commercially useful, but there is the possibility of reconstructing pathways pioneered with isolated recombinant enzymes in cells. It should also be noted that the individual reactions are unoptimized, hence there is likely for considerable scope for improved yields, especially in cells.
We hope that the results will promote further work on the construction of hybrid natural/unnatural pathways for the synthesis of useful antibacterials, especially carbapenems and related compounds, in a manner that is more complex, but the same in concept as that applied very so successfully for the production of clinically useful penicillins many decades ago. ${ }^{2}$ Modern molecular and synthetic biology approaches coupled to structural and mechanistic insights should enable construction of more complex pathways than the simple, though hugely important, penicillin side-chain exchange process. $^{42}$

More generally the results further highlight the largely unexplored biocatalytic potential of crotonase superfamily enzymes. ${ }^{14,43}$ In this regard, the capability to make compounds with 'demanding' quaternary centres is striking. This is exemplified in the ThnE W124F/V153M-catalysed reaction of (2S)-5-methyl-GHP and C2 methylmalonyl-CoA to give $(5 S, 6 S)$-5,6-dimethyl- $t$-CMP. However, at least for the currently available variants, there are limitations in substrate scope, e.g. we did not observe formation of $(5 S, 6 R)$-5,6-dimethyl-t-CMP, likely due to steric constraints (a potential clash between the trisubstituted $(Z)$-enolate intermediate, resulting from decarboxylation of $(2 S)$-methylmalonyl-CoA, and (2S)-5-methyl-GHP, Fig. 2D). The results reveal that the steric limitations are not as tight for reactions with the (2S)-3-methylGHP isomers where both C-6 epimers were observed, though varying in ratios depending on the CMPS variant used (Table S2 and S3, Fig. 3). We have previously found that CMPS variants with a $\beta$-branched residue (i.e. Val or Ile) at the oxyanion hole forming residue $108_{\text {CarB }}$ (wild-type Met)/153 ThnE (wild-type Val) favor the 
formation of products with the $(6 R)$-stereochemistry, ${ }^{24,26}$ as is also the case with formation of the dialkylated products (e.g. entries 2 and 3 in Table S2 and entry 3 in Table S3). On the other hand, CMPS variants with a non- $\beta$-branched residue (Leu, Met, Ala) have a bias towards producing the $(6 S)$-epimer of 3,6-dimethyl- $t$ CMP (e.g. entries 7-9 in Table S2 and entries 1, 2, 8 and 9 in Table S3). These preferences may result from a steric clash between the methyl-substituent on the trisubstituted $(E)$-enolate, resulting from $(2 R)$-methylmalonyl-CoA decarboxylation, and the $\beta$ branched residues (Val or Ile) at the oxyanion hole forming residue of tested CMPS variants (Fig. 2E). ${ }^{26}$ It is also notable that, in general, Trp79 ${ }_{\mathrm{CarB}} / \operatorname{Trp} 124_{\mathrm{ThnE}}$-based CMPS variants with less bulky residues at this position, favor formation of $(6 S)$ - epimers (entries 4 and 5 in Table S2 and entries 5-7 in Table S3), consistent with earlier reports. ${ }^{24,26}$ This is likely due, at least in part, to provision of more space in a hydrophobic region with consequently improved accommodation of the $(2 R)$-alkyl group of the malonyl-CoA derivative and/or the subsequently formed $(E)$ enolate. $^{24,26}$

Although CarA engineering/redesign was not our main focus, combined with previous reports, ${ }^{21,25,28,44}$ our results highlight the potential of synthetases for stereoselective biocatalysis of $\beta$ lactams. $^{2}$ Notably, whilst CarA demonstrated a considerable degree of promiscuity, not all substrate analogues were converted, suggesting scope for structure- and analogue-guided engineering to expand the scope of Car A catalysis, including with respect to making C-5 alkyl-carbapenams. Since crystal structures of CarA in complex with its acyl-adenylate $t$-CMP intermediate (or analogue thereof) are not yet available, ${ }^{32}$ the substrate analogue studies described here may help in mapping the CarA active site topology, with the aim of identifying CarA variants for efficient production of substituted carbapenams.

\section{AUTHOR INFORMATION}

\section{Corresponding Author}

christopher.schofield@chem.ox.ac.uk

\section{Author Contributions}

R.B.H. and C.J.S. designed the study and wrote the manuscript. L.H. synthesized and characterized the protected form of the GHP derivatives. R.B.H. prepared and characterized CMPSs and CarA, carried out enzyme assays, analyzed the data, purified the products of enzyme catalysis, and ran NMR analyses. R.B.H. and T.D.W.C. analyzed the NMR data. All authors commented on the results and approved the final version of the manuscript.

\section{Funding Sources}

Biotechnology and Biological Sciences Research Council and Medical Research Council.

\section{ASSOCIATED CONTENT}

Supporting Information. Experimental procedures and compound characterization data. This material is available free of charge via the Internet at http://pubs.acs.org.

\section{REFERENCES}

1. Butler, M. S.; Blaskovich, M. A.; Cooper, M. A., J. Antibiot. 2013, 66, 571-591.

2. Hamed, R. B.; Gomez-Castellanos, J. R.; Henry, L.; Ducho, C.; McDonough, M. A.; Schofield, C. J., Nat. Prod. Rep. 2013, 30, 21-107.
3. Shih, D. H.; Baker, F.; Cama, L.; Christensen, B. G., Heterocycles 1984, 21, 29-40.

4. Shih, D. H.; Cama, L.; Christensen, B. G., Tetrahedron Lett. 1985, 26, 587-590.

5. Dürckheimer, W.; Blumbach, J.; Lattrell, R.; Scheunemann, K. H., Angew. Chem. Int. Ed. 1985, 24, 180-202.

6. Hamilton-Miller, J., J. Antimicrob. Chemother. 1999, 44, 729734.

7. $\quad$ Earl, J.; Kirkpatrick, P., Nat. Rev. Drug Discov. 2003, 2, 97-98.

8. Rothstein, J.; Patel, S.; Regan, M.; Haenggeli, C.; Huang, Y.; Bergles, D.; Jin, L.; Hoberg, M.; Vidensky, S.; Chung, D., Nature 2005, 433, $73-77$.

9. Panday, S. K., Tetrahedron-Asymmetry2011, 22, 1817-1847.

10. List, B., Tetrahedron 2002, 58, 5573-5590.

11. Li, R. F.; Stapon, A.; Blanchfield, J. T.; Townsend, C. A., J. Am. Chem. Soc. 2000, 122, 9296-9297.

12. McGowan, S. J.; Sebaihia, M.; O'Leary, S.; Hardie, K. R.; Williams, P.; Stewart, G. S. A. B.; Bycroft, B. W.; Salmond, G. P. C., Mol. Microbiol. 1997, 26, 545-556.

13. Nunez, L. E.; Mendez, C.; Brana, A. F.; Blanco, G.; Salas, J. A., Chem. Biol. 2003, 10, 301-311.

14. Hamed, R. B.; Batchelar, E. T.; Clifton, I. J.; Schofield, C. J., Cell. Mol. Life Sci. 2008, 65, 2507-2527.

15. Batchelar, E. T.; Hamed, R. B.; Ducho, C.; Claridge, T. D. W.; Edelmann, M. J.; Kessler, B.; Schofield, C. J., Angew. Chem. Int. Ed. Engl. 2008, 47, 9322-5.

16. Gerratana, B.; Arnett, S. O.; Stapon, A.; Townsend, C. A., Biochemistry 2004, 43, 15936-15945.

17. Sleeman, M. C.; Schofield, C. J., J. Biol. Chem. 2004, 279, 6730-6736.

18. Sleeman, M. C.; Sorensen, J. L.; Batchelar, E. T.; McDonough, M. A.; Schofield, C. J., J. Biol. Chem. 2005, 280, 34956-34965.

19. Hamed, R. B.; Batchelar, E. T.; Mecinovic, J.; Claridge, T. D. W.; Schofield, C. J., ChemBioChem 2009, 10, 246-250.

20. Bodner, M. J.; Li, R.; Phelan, R. M.; Freeman, M. F.; Moshos, K. A.; Lloyd, E. P.; Townsend, C. A., ChemBioChem 2011, 12, 2159-65.

21. Gerratana, B.; Stapon, A.; Townsend, C. A., Biochemistry 2003, 42, 7836-7847.

22. Clifton, I.J.; Doan, L. X.; Sleeman, M. C.; Topf, M.; Suzuki, H.; Wilmouth, R. C.; Schofield, C. J., J. Biol. Chem. 2003, 278, 20843-20850.

23. Chang, W. C.; Guo, Y.; Wang, C.; Butch, S. E.; Rosenzweig, A. C.; Boal, A. K.; Krebs, C.; Bollinger, J. M., Jr., Science 2014, 343, 1140-4.

24. Hamed, R. B.; Henry, L.; Gomez-Castellanos, J. R.; Asghar, A.; Brem, J.; Claridge, T. D. W.; Schofield, C. J., Org. Biomol. Chem. 2013, 11, 8191 .

25. Hamed, R. B.; Henry, L.; Gomez-Castellanos, J. R.; Mecinović, J.; Ducho, C.; Sorensen, J. L.; Claridge, T. D. W.; Schofield, C. J., J. Am. Chem. Soc. 2012, 134, 471-479.

26. Hamed, R. B.; Gomez-Castellanos, J. R.; Thalhammer, A.; Harding, D.; Ducho, C.; Claridge, T. D. W.; Schofield, C. J., Nat. Chem. 2011, 3,365-71.

27. Hamed, R. B.; Gomez-Castellanos, J. R.; Sean Froese, D.; Krysztofinska, E.; Yue, W. W.; Schofield, C. J., ChemBioChem 2016, 17, 471-3.

28. Hamed, R. B.; Mecinovic, J.; Ducho, C.; Claridge, T. D. W.; Schofield, C. J., Chem. Commun. 2010, 46, 1413-1415.

29. Sorensen, J. L.; Sleeman, M. C.; Schofield, C. J., Chem. Commun. 2005, 1155-1157.

30. Erb, T. J.; Berg, I. A.; Brecht, V.; Muller, M.; Fuchs, G.; Alber, B. E., Proc. Natl. Acad. Sci. U. S. A. 2007, 104, 10631-10636.

31. Repka, L. M.; Reisman, S. E., J. Org. Chem. 2013, 78, 12314 12320 .

32. Miller, M. T.; Gerratana, B.; Stapon, A.; Townsend, C. A.; Rosenzweig, A. C., J. Biol. Chem. 2003, 278, 40996-41002.

33. Miller, M. T.; Bachmann, B. O.; Townsend, C. A.; Rosenzweig, A. C., Proc. Natl. Acad. Sci. U. S. A. 2002, 99, 14752-14757.

34. Raber, M. L.; Castillo, A.; Greer, A.; Townsend, C. A., ChemBioChem 2009, 10, 2904-2912. 
35. Raber, M. L.; Arnett, S. O.; Townsend, C. A., Biochemistry 2009, 48, 4959-4971.

36. Albers-Schönberg, G.; Arison, B. H.; Hensens, O. D.; Hirshfield, J.; Hoogsteen, K.; Kaczka, E. A.; Rhodes, R. E.; Kahan, J. S.; Kahan, F. M.; Ratcliffe, R. W.; Walton, E.; Ruswinkle, L. J.; Morin, R. B.; Christensen, B. G., J. Am. Chem. Soc. 1978, 100, 6491-6499.

37. Parker, W. L.; Rathnum, M. L., J. Antibiot. 1982, 35, 300-5.

38. Haneishi, T.; Nakajima, M.; Serizawa, N.; Inukai, M.; Takiguchi, Y.; Arai, M.; Satoh, S.; Kuwano, H.; Tamura, C., J. Antibiot. 1983, 36, 1581-4.

39. Yoshioka, T.; Watanabe, A.; Kojima, I.; Shimauchi, Y.; Okabe, M.; Fukagawa, Y.; Ishikura, T., J. Antibiot. 1984, 37, 211-7.
40. Bycroft, B. W.; Maslen, C.; Box, S. J.; Brown, A. G.; Tyler, J. W., J. Chem. Soc., Chem. Commun. 1987, 1623-1625.

41. Bycroft, B. W.; Maslen, C.; Box, S. J.; Brown, A.; Tyler, J. W., J. Antibiot. 1988, 41, 1231-1242.

42. Behrens, O. K.; Corse, J.; Edwards, J. P.; Garrison, L.; Jones, R G.; Soper, Q. F.; Van Abeele, F. R.; Whitehead, C. W., J. Biol. Chem. 1948, 175, 771-792.

43. Grogan, G.; Graf, J.; Jones, A.; Parsons, S.; Turner, N. J.; Flitsch, S. L., Angew. Chem. Int. Ed. 2001, 113, 1111-1114.

44. Sleeman, M. C.; MacKinnon, C. H.; Hewitson, K. S.; Schofield, C. J., Bioorg. Med. Chem. Lett. 2002, 12, 597-599.

\section{Table of Contents artwork}

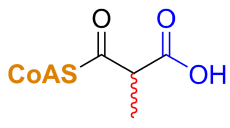

alkylmalonyl-CoA

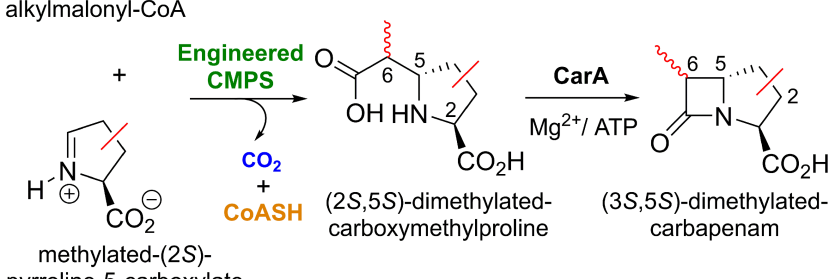

pyrroline-5-carboxylate 